\section{Estudo cefalométrico comparativo entre respiradores nasais e predominantemente bucais}

\author{
Jussara Marinho Dias Frasson ${ }^{1}$, Maria Beatriz Borges \\ de Araújo Magnani², Darcy Flávio Nouer ${ }^{3}$, Vânia Célia \\ Vieira de Siqueira ${ }^{4}$, Nádia Lunardi ${ }^{5}$
}

\title{
Comparative Cephalometric Study Between Nasal and Predominantly Mouth Breathers
}

Palavras-chave: função naso-respiratória, morfologia dentofacial, ortodontia.

Key words: Nasorespiratory Function, Dentofacial Morphology, Orthodontics.

\section{Resumo / Summary}

$\mathrm{O}$ bjetivo: avaliar a possível influência do padrão respiratório na determinação das dimensões craniofaciais, tendo como base a análise cefalométrica de Tweed-Merrifield, acrescidas do ângulo SN-GoGn e do ângulo do eixo Y. Metodologia: A amostra selecionada para o presente estudo constou de 50 telerradiografias, tomadas em norma lateral e posição natural de cabeça, de jovens do sexo feminino, na faixa etária de 9 a 12 anos (idade média de 10 anos e 5 meses) com maloclusão de Classe I. Após o diagnóstico do padrão respiratório, dividiu-se a amostra em dois grupos, assim constituídos: grupo controle - 25 telerradiografias de respiradores nasais e grupo experimental - 25 telerradiografias de respiradores predominantemente bucais. Resultados: foram submetidos à análise descritiva (média e desvio padrão), teste F e teste "t" de Student com nível de significância de 5\%, através dos quais foi possível constatar não existir diferença significativa entre os grupos com respiração nasal quando comparado com o grupo de respiração predominantemente bucal para nenhumas das grandezas estudadas.

\begin{abstract}
A : to evaluate the possible correlation between the respiratory pattern in determining the craniofacial dimensions, using as baseline the Tweed-Merrifield's cephalometric analysis, added to angle SN-GoGn and to $\mathrm{Y}$ axis angle. Methodology: The selected sample to this study comprised 50 teleradiographies taken in lateral and natural positions of the head in young female patients at the age of 9 to 12 years, presenting mean age of 10 years and 5 months and Class 1 malocclusion. After diagnosis of respiratory pattern, the sample was divided into two groups: control group, 25 teleradiographies of nasal breathers in lateral and natural positions of the head; experimental group, 25 teleradiographies of predominantly mouth breathers in lateral and natural positions of the head. Results: The results were submitted to descriptive analysis (mean and standard deviation), test F and "t" Student test with significance level of $5 \%$. There was no significant difference between the group with nasal breathing and the group with predominantly mouth breathing for any of the studied variables.
\end{abstract}

${ }^{1}$ Especialista em Odontopediatria, Ortodontia e Ortopedia Facial. Mestre em Ortodontia. Doutoranda em Fisiologia. Odontopediatra, Ortodontista e Professora Convidada de Ortodontia.

${ }^{2}$ Professora Assistente MS-3. Sub-coordenadora da Disciplina de Ortodontia da FOP-Unicamp.

${ }^{3}$ Professor Titular da Disciplina de Ortodontia da FOP-Unicamp. Coordenador de Pós-Graduação de Mestrado e Doutorado em Ortodontia. ${ }^{4}$ Professora Assistente Doutora do Curso de Pós Graduação em Ortodontia Mestre em Ortodontia. Professora Convidada. FOP-UNICAMP

Endereço para correspondência: R. Prudente de Moraes 1395 ap. 92 Piracicaba SP 13416-720. Este artigo foi submetido no SGP (Sistema de Gestão de Publicações) da RBORL em 13 de agosto de 2005. Artigo aceito em 21 de setembro de 2005. 


\section{INTRODUÇÃO}

A relação da função respiratória com o desenvolvimento da oclusão e da morfologia craniofacial revela uma longa e controvertida história no âmbito da ortodontia.

A preocupação quanto às possíveis alterações esqueléticas e dentárias decorrentes do padrão respiratório desperta interesse a bastante tempo nas áreas de ortodontia, de otorrinolaringologia e da fonoaudiologia, entre outras. Cheng et al. (1988), Cooper (1989) $)^{1}$, Parolo \& Bianchini (2000), Queluz \& Gimenez (2000) ${ }^{2}$, concluíram que a abordagem interdisciplinar da obstrução nasal, envolvendo otorrinolaringologistas e ortodontistas, é vantajosa para que se reduza o efeito adverso da alteração do modo respiratório na oclusão e na morfologia craniofacial.

A respiração bucal possui uma etiologia multifatorial que pode variar tanto de uma predisposição anatômica (passagem aérea estreita) como obstruções físicas, tais como: hipertrofia das tonsilas palatinas, hipertrofia das adenóides, pólipos nasais, desvios de septo nasal, alergias respiratórias, condições climáticas, sinusites, hipertrofias de cornetos, posição de dormir, aleitamento artificial, ou decorrente de hábitos bucais deletérios, como sucção digital ou de chupeta, que dependendo da intensidade, da freqüência e da duração do hábito deformam a arcada dentária e alteram todo o equilíbrio facial (Andrade \& Majolo, 20003; Rodrigues \& Rodrigues, 2003).

Mocellin (1992)4 avaliou a relação da respiração bucal com o desenvolvimento dentofacial, alertando para o fato de que nos primeiros 10 anos de vida ocorre o maior desenvolvimento da face. O autor afirmou que todo paciente com obstrução nasal crônica pode se tornar um respirador bucal, sendo a obstrução mais freqüente a hipertrofia de adenóides.

Autores como: Linder-Aronson \& Bäckström (1960), Ricketts (1968), Hawkins (1969)5, Paul \& Nanda (1973), Carbone \& Bernaba (1977), Linder-Aronson (1979) ${ }^{6}$, McNamara Jr. $(1981)^{7}$, Bresolin et al. $(1983)^{8}$, Melsen et al. (1987)9 , Martinez Esteinou \& Omana Vidal (1988), Cheng et al. (1988), Martins (1988), Yamada et al. (1997) ${ }^{10}$, Fujiki \& Rossato (1999) ${ }^{11}$, Sabatoski (1999) $)^{12}$, Bizetto (2000) ${ }^{13}$, Motonaga et al. (2000) $)^{14}$, Mello (2001), Pereira et al. (2001), Simas Netta et al. (2004) ${ }^{15}$, relacionaram de uma forma direta, a obstrução das vias aéreas, a permanência dessa obstrução e o desenvolvimento do complexo craniofacial. Acredita-se que a obstrução prolongada traz como conseqüência o surgimento de um quadro de respiração bucal, conduzindo a uma alteração desfavorável no complexo craniofacial, ocasionando o desenvolvimento de um conjunto de modificações funcionais, dento-alveolares e esqueléticas, atribuídas aos indivíduos, denominados de síndrome da face longa. Tal síndrome se caracteriza por apresentar-se clinicamente com mordida aberta postural, narina estreita e subdesenvolvida, lábio superior curto, vestíbulo-versão de incisivos superiores, lábio inferior evertido, expressão facial vaga, arco maxilar estreito, em forma de "V", palato profundo e maloclusão de Classe II e à presença de mordida cruzada, tonsilas faríngeas e palatinas hipertróficas, mordida aberta anterior, interposição lingual (Ricketts, 1968).

Considera-se como respirador bucal aquele que possui um desvio do padrão de respiração nasal normal, sendo esta, por vezes, insuficiente; portanto, passa a ser substituída pela suplência bucal ou mista (Lusvarghi, $1999)^{16}$.

Segundo Bueno Jr. (1996), a exclusão completa do nariz da respiração leva, em longo prazo, a alterações mucosas profundas. A obstrução mecânica no interior do nariz, como acontece no desvio de septo, na hipertrofia dos cornetos ou em estenoses cicatriciais, pode ocasionar a respiração pela boca e suas conseqüências e, também, pode causar doenças da mucosa do nariz e dos seios paranasais.

A causa mais comum da respiração bucal é, sem dúvida, a obstrução nasal, sendo mais importante ainda quando se trata de crianças, por estarem em crescimento e desenvolvimento. Uma pessoa nunca é exclusivamente respiradora bucal; o que existe é a respiração predominantemente bucal ou mista (Lusvarghi, 1999) ${ }^{16}$.

A obstrução nasal pode ser dividida quanto à lateralidade (uni ou bilaterais) e quanto ao tempo (intermitente e constante). Casos de obstrução nasal unilateral e constante podem ter como origem desvio de septo, corpos estranhos, tumores, pólipos, imperfuração coanal bilateral e abscessos septais (Klein, 1987) ${ }^{17}$.

A relação da respiração bucal e desenvolvimento de maloclusões é, contudo, muito polêmica pois alguns autores não relacionam a obstrução nasal como fator primário de maloclusão, e sim um fator de desequilíbrio muscular entre a musculatura interna (língua que está posicionada mais anterior e inferior) e excesso de pressão da musculatura das bochechas sobre a maxila, ficando o arco superior em forma de "V" em pacientes com adenóides volumosas, que levariam ao comprometimento da estabilidade do tratamento ortodôntico (Quick \& Gundlach $(1978)^{18}$, Diamond $(1980)^{19}$, Subtelny $(1980)^{20}$, Vig et al. (1981) ${ }^{21}$, O'Ryan et al., (1982)22, Bressolin et al. (1984), Santos-Pinto \& Monnerat (1986) ${ }^{23}$, Klein (1986) ${ }^{24}$, Meredith (1988) ${ }^{25}$, Cooper (1989) ${ }^{1}$, Smith \& Gonzales $(1989)^{26}$, Tourne $(1990)^{27}$, Fields et al. $(1991)^{28}$, Woodside et al. (1991), Vig (1998), Castilho et al. (2002) ${ }^{29}$, Solow \& Greve, 1980) ${ }^{30}$.

Autores tais como: Watson et al. (1968), Muñoz (1970), Quick \& Gundlach (1978) ${ }^{18}$, Diamond $(1980)^{19}$, Subtelny $(1980)^{20}$, Vig et al. (1981) ${ }^{21}$, O'Ryan et al., (1982) $)^{22}$, Bressolin et al. (1984), Santos-Pinto \& Monnerat (1986) ${ }^{23}$, Klein $(1986)^{24}$, Meredith $(1988)^{25}$, Cooper $(1989)^{1}$, Smith \& Gonzales (1989) ${ }^{26}$, Tourne $(1990)^{27}$, Fields et al. $(1991)^{28}$, 
Woodside et al. (1991), Vig (1998), Castilho et al. (2002)29, não encontraram evidências diretas da relação do padrão respiratório com maloclusões.

Muitos estudos relacionam, no respirador bucal, a postura da cabeça e do pescoço com a postura de todo o corpo. A postura estendida da cabeça causa uma modificação dos diversos elementos anatômicos móveis entre a cabeça e o pescoço, como o aumento da distância entre o occipital e o arco dorsal da primeira vértebra cervical. Esta posição rotaciona a cabeça para cima, facilitando a passagem do ar pela bucofaringe, facilitando a adaptação do sistema músculo-esquelético, que tende a adaptar-se por uma alteração postural e funcional também dos lábios, língua, musculatura mastigatória, mandíbula, palato mole e musculatura ocular (Rahal \& Krakauer, 2001, Harvold et al. (1973) ${ }^{31}$, Koski \& Lähdemäki (1975), Rubin (1980), Harvold et al. (1981) $)^{32}$, Weber et al. (1981) ${ }^{33}$, Miller et al. $(1984)^{34}$, Tarvonen \& Koski (1987), Tourne (1990) $)^{27}$, Jabur et al. (1997), Para Mocellin \& Ciuffi (1997), Jorge (2001) ${ }^{35}$, Simas Netta et al. (2004) $)^{15}$.

O'Ryan et al. (1982)22, numa revisão da literatura, estudaram a relação entre a função respiratória e a morfologia dentofacial. Apesar de inúmeros trabalhos sugerirem uma relação direta de causa e efeito entre a obstrução das vias aéreas nasais e as alterações dentofaciais, os autores concluíram que existe uma necessidade de estudos que avaliem, quantitativa e longitudinalmente, os fluxos de ar nasal e bucal durante a respiração, antes de se atribuir a obstrução respiratória à etiologia no desenvolvimento de uma deformidade dentofacial específica.

Uma relação direta de causa e efeito entre a obstrução nasal e bucal e a morfologia dentofacial alterada e um diagnóstico preciso do padrão respiratório são necessários para que a obstrução aérea possa ser indicada como significante fator etiológico no desenvolvimento de alguma deformidade dentofacial específica, Schulhof (1978), Ianni Filho et al. (2001) ${ }^{36}$.

Os estudos da função respiratória nasal devem ser objetivos, utilizando-se de exames precisos para a avaliação do modo respiratório. Alguns utilizam a anamnese e o exame clínico, como Massler \& Zwemer $(1953)^{37}$, e testes clínicos, como Quinn $(1983)^{38}$. Outros preconizam o uso de uma mecha de algodão colocada abaixo do nariz e/ou espelhos colocados alternadamente na frente do nariz durante a respiração.

A cefalometria é utilizada para analisar o grau de obstrução na bucofaringe e na nasofaringe, como McNamara Jr. (1984).

A rinomanometria, associada à anamnese e a exames clínicos, também é utilizada para quantificar a resistência respiratória nasal (Thuer et al., 1989) ${ }^{39}$.

A videoendoscopia tem sido considerada um método revolucionário de diagnóstico, pois com a utilização de telescópios flexíveis ou rígidos consegue-se uma visuali- zação direta das tonsilas palatinas, cavidade nasal, tonsilas faringianas e do tamanho do espaço livre na entrada da nasofaringe. Quanto à avaliação adequada do real grau de obstrução nasal, através de técnicas que correspondam à realidade, permitindo a instituição de terapia adequada, a nasofibroendoscopia se mostrou superior à radiografia lateral do cavum faríngeo na avaliação da obstrução nasofaringeana (Chami, 1998) ${ }^{40}$.

Em virtude da importância em se estudar a respiração predominantemente bucal e suas possíveis repercussões no complexo craniofacial, objetivou-se no presente estudo comparar cefalometricamente jovens com diferentes padrões respiratórios, nos seguintes aspectos:

a) o padrão de crescimento craniofacial;

b) o perfil facial;

c) o relacionamento das bases apicais com o crânio entre si;

d) as alturas faciais anteriores, posteriores e o índice da altura facial;

e) verificar a ocorrência ou não, de diferenças entre os grupos respiradores nasais e predominantemente bucais.

\section{MATERIAL E MÉTODO}

\section{Seleção da amostra}

Este trabalho somente iniciou-se após a aprovação pelo Comitê de Ética em Pesquisa em Seres Humanos (CEP), da FOP-UNICAMP, conforme documentação exigida pela Resolução 196/96 da Comissão Nacional de ética em Pesquisa (CONEP) do Conselho Nacional de Saúde do Ministério da Saúde.

O material utilizado no presente estudo constou de telerradiografias do acervo do arquivo de documentação ortodôntica da Área de Ortodontia, do Departamento de Odontologia Infantil da FOP/UNICAMP, sendo selecionadas 50 telerradiografias, tomadas em norma lateral e na posição natural da cabeça, de 50 crianças brasileiras, leucodermas, do sexo feminino, na faixa etária compreendida entre 9 e 12 anos, de escolas de primeiro grau da rede estadual de ensino público da cidade de Limeira.

Empregou-se também o laudo obtido pelo otorrinolaringologista, dividindo a amostra em grupo controle, respiradores nasais $(n=25)$ e grupo experimental, respiradores predominantemente bucais $(n=25)$.

\section{Critério para obtenção e seleção da amostra}

Para a seleção da amostra do presente estudo foram seguidos os critérios constantes no Quadro 1:

\section{Exame de Nasofibroscopia}

Todas as crianças foram submetidas a uma avaliação previamente ao estudo por um médico Otorrinolaringologista, responsável pelo diagnóstico do padrão respiratório. 
Quadro 1. Critérios adotados para seleção da amostra.

\begin{tabular}{|c|c|}
\hline Critérios de inclusão & Critérios de exclusão \\
\hline - Jovens do sexo feminino & - Jovens do sexo masculino \\
\hline $\begin{array}{l}\text { - Jovens com chave de molar em } \\
\text { Classe I, de Angle e dentição mista. }\end{array}$ & $\begin{array}{l}\text { - Jovens com maloclusão } \\
\text { de Classe II e Classe III }\end{array}$ \\
\hline $\begin{array}{l}\text { - Ausência de tratamento ortodôn- } \\
\text { tico e/ou ortopédico funcional dos } \\
\text { maxilares. }\end{array}$ & $\begin{array}{l}\text { - Jovens que estavam ou } \\
\text { estiveram com tratamento } \\
\text { ortodôntico }\end{array}$ \\
\hline - Ausência de lesões cariosas. & $\begin{array}{l}\text { - Jovens com lesões cari- } \\
\text { osas extensas. }\end{array}$ \\
\hline $\begin{array}{l}\text { - Nitidez e contrastes suficientes } \\
\text { para uma boa visualização e } \\
\text { identificação das estruturas que } \\
\text { compõem o tecido tegumentar, as } \\
\text { estruturas ósseas e os elementos } \\
\text { dentários. }\end{array}$ & $\begin{array}{l}\text { - Telerradiografias sem } \\
\text { nitidez e sem contraste, }\end{array}$ \\
\hline $\begin{array}{l}\text { - Ausência de distorções das radi- } \\
\text { ografias }\end{array}$ & $\begin{array}{l}\text { - telerradiografias com } \\
\text { distorções de imagens. }\end{array}$ \\
\hline
\end{tabular}

Foram examinadas a garganta o e nariz por meio de exame clínico e nasofibroscopia. O otorrinolaringologista avaliou o questionário respondido pelos pais, a anamnese preenchida pela pesquisadora, bem como a telerradiografia, tomada em norma lateral e em posição natural de cabeça, cujos elementos participaram do processo de diagnóstico do padrão respiratório, classificando-os em padrão respiratório clinicamente normal ou com respiração predominantemente bucal.

O otorrinolaringologista utilizou o protocolo de diagnóstico do padrão respiratório baseado nos trabalhos de Wang et al. (1997), Ianni Filho $(2001)^{36}$ e os resultados da vídeo-endoscopia para emitir o laudo final do padrão respiratório de cada criança examinada. O laudo do diagnóstico do padrão respiratório e as gravações em videocassete do exame de nasofibroscopia óptica passaram a fazer parte do acervo da Disciplina de Ortodontia do Departamento de Odontologia Infantil da FOP/UNICAMP.

\section{Método Radiográfico}

Realizaram-se telerradiografias, tomadas em norma lateral e em posição natural de cabeça, sempre pelo mesmo operador, técnico responsável do Serviço de Documentação Odontológica, seguindo as normas estabelecidas pela Faculdade de Odontologia de Piracicaba - UNICAMP.

Conforme dados do arquivo dos pacientes, para a seleção da amostra, as crianças foram instruídas a manterem seus dentes em oclusão habitual, lábios relaxados e se posicionarem confortavelmente em pé, olhando para o espelho situado a $1 \mathrm{~m}$ de distância. Antes de adequar o paciente no cefalostato, a posição do corpo e da cabeça foram ensaiadas e repetidas se necessário, de tal maneira que as pupilas estivessem no centro dos olhos.

As olivas foram então posicionadas no conduto auditivo externo exercendo a mínima pressão.

Confirmada a posição da cabeça pelo próprio paciente frente a frente, o apoio nasal foi colocado no Násio, contatando levemente a pele, apenas para estabelecer apoio no plano vertical, prevenindo qualquer movimentação da cabeça.

As crianças foram orientadas para que permanecessem com os dentes em oclusão habitual e lábios relaxados. Para evitar falsa impressão de obstrução nasofaringeana, solicitou-se à criança que deglutisse antes da tomada radiográfica onde o lado direito da face do paciente posicionou-se mais próximo do filme radiográfico.

Foram utilizados métodos para a proteção do paciente e do operador, tais como o uso de avental de chumbo para absorver a radiação secundária.

O aparelho utilizado foi o Raios-X Raiograph Plus, fabricante: Villa Sistemi Medicali \& r.l. (Italy), apresentando filtragem total equivalente a $2,5 \mathrm{~mm}$ e o tamanho do foco com $0,6 \mathrm{~mm}^{\prime} 0,6 \mathrm{~mm}$.

Foi calibrado para operar com $16 \mathrm{~mA}$ e $77 \mathrm{KV}$, com um tempo de exposição de 0,4 a 0,5 segundos dependendo da massa corpórea da criança.

O filme utilizado foi o T-MAT G/RA-1(Kodak Brasileira Com. e Ind. Ltda. -S.J. Campos, SP, Brasil), com uma dimensão de $18 \mathrm{~cm}^{-} 24 \mathrm{~cm}$ e o ecran foi Lanex XOMATIC Médium..

Para a revelação, foi utilizada a processadora automática Revel (X-Tec-processadora de Raio X Ltda. Me, Brasil), com revelador e fixador reforçados RP X-OMATI (Kodak Brasileira Com. e Ind. Ltda., S.J. Campos, SP, Brasil).

Para evidenciar os tecidos moles do perfil facial, foi utilizado um filtro de alumínio, posicionado junto ao colimador de feixe de radiação.

O coeficiente de distorção apresentado foi de 10\% em média.

Utilizaram as grandezas esqueléticas da análise cefalométrica, de Merrifield \& Klontz (1993), e incluídas as mensurações, do ângulo SN-GoGn e do ângulo do eixo Y. Portanto, foram avaliadas três variáveis lineares e oito angulares. Obtiveram-se dois cefalogramas para cada telerradiografia, pela mesma pesquisadora, em uma sala obscurecida e sobre o negatoscópio da Faculdade de Odontologia de Piracicaba/UNICAMP, não obedecendo à ordem numérica estabelecida inicialmente para os pacientes e reavaliada com intervalo de uma semana. Determinaram-se os valores pelas médias destes valores.

\section{Avaliação cefalométrica}

A delimitação das estruturas anatômicas foi feita conforme o protocolo de traçado cefalométrico do curso de Ortodontia da FOP/Unicamp, (segundo Krogman \& Sassouni, 1957; Interlandi, 1968; Vion, 1994) sendo considerada a imagem menor, correspondente ao lado mais 
próximo do filme, com menor distorção, A demarcação dos pontos cefalométricos e o estabelecimento dos traçados de orientação tiveram com referência os trabalhos de Steiner (1953), Krogman \& Sassouni (1957)41, Interlandi (1968) e Horn (1992), conforme Figura 1.

a) Sela (S) - centro geométrico da sela túrcica, determinado por inspeção;

b) Násio (N) - intersecção da sutura internasal com a sutura násio-frontal, no plano sagital mediano, determinado por inspeção.

c) Pório (Po) - ponto mais superior do contorno do meato acústico externo. Sua identificação é bastante difícil em virtude da superposição de outros elementos anatômicos. Para a sua localização, utilizaram-se os referenciais propostos por Miyashita (1996), onde o meato acústico externo está localizado posteriormente ao processo condilar da mandíbula, acima do Básio e do processo odontóide do axis;

d) Orbitário (Or) - ponto mais inferior do contorno da cavidade orbitária direita;

e) Espinha Nasal Anterior (ENA) - ponto mediano formado pelo prolongamento das duas maxilas na imagem inferior e anterior do assoalho nasal;

f) Espinha Nasal Posterior (ENP) - ponto mediano formado pela união das bordas posteriores dos dois ossos palatinos;

g) A (Subespinhal) - ponto mais profundo da concavidade anterior da maxila, entre a espinha nasal anterior e o limite alveolar do arco dentário superior;

h) B (Supramentoniano) - ponto mais profundo da concavidade anterior da sínfise;

i) Pogônio (Pg) - ponto mais anterior da sínfise mandibular;

j) Pogônio tegumentar (Pgt) - ponto mais anterior ou proeminente do tecido mole do queixo, no plano sagital mediano;

k) Mentoniano (Me) - ponto mais inferior do contorno da sínfise mandibular;

1) Gnátio (Gn) - ponto mais anterior e inferior da sínfise mandibular;

m) Articular (Ar) - ponto de interseção do contorno externo da base craniana com o processo condilar da mandíbula;

n) Gônio (Go) - ponto mais posterior e inferior da mandíbula no sentido ântero-posterior. Situado na bissetriz do ângulo formado pela tangente da borda posterior do ramo com a tangente da borda inferior da mandíbula;

o) Ápice radicular inferior (Ari) - ponto na região mais inferior do ápice radicular do incisivo central inferior;

p) Borda incisal inferior (BII) - ponto na região mais superior da borda da coroa do incisivo central inferior;

\section{Demarcação dos planos e linhas}

Após a identificação dos pontos cefalométricos, traçaram-se planos e linhas, conforme Figura 2.

\section{Mensuração das grandezas lineares}

Altura facial Posterior (AFP) - distância do ponto Ar ao plano mandibular, tangenciando a borda posterior do ramo da mandíbula (Merrifield, 1989).

Altura facial Anterior (AFA) - distância entre o ponto Me e sua projeção ortogonal sobre o Plano Palatino. O plano Palatino é traçado unindo os pontos ENP e ENA (Merrifield, 1989).

Índice de Altura Facial (IAF) - determinado pela razão da AFP pela AFA (Horn, 1992).

\section{Mensuração das grandezas angulares}

a) FMA - ângulo formado pela interseção do plano Horizontal de Frankfurt (PHF) com o Mandibular (MD). O plano Horizontal de Frankfurt foi traçado pela união dos pontos Po e Or. O plano Mandibular pela tangente à borda inferior do corpo da mandíbula, passando pelo ponto Me (Tweed; 1954).

b) SNA - ângulo formado pela interseção das linhas Sela-Násio e Násio-A (Riedel; 1952).

c) SNB - ângulo determinado pela interseção das linhas Sela-Násio e Násio-B (Riedel; 1952).

d) ANB - ângulo determinado pela interseção das linhas Násio-A e Násio-B (Riedel; 1952).

e) Ângulo Z - determinado pela interseção do plano Horizontal de Frankfurt com a linha que tangencia o ponto Pg e o lábio mais anterior (Merrifield; 1966).

f) Ângulo do eixo $Y$ - determinado pelo eixo $Y$, que parte do ponto (S) ao ponto Gn, com o plano Horizontal de Frankfurt (Downs; 1948).

g) Ângulo SN-GoGn - determinado pela interseção do plano mandibular (GoGn) com a linha S-N.

h) Ângulo IMPA - interseção do plano mandibular com o longo eixo do incisivo central inferior.

\section{Análise estatística}

O planejamento estatístico desta pesquisa foi realizado em duas etapas: a primeira correspondendo ao cálculo de erro, que determina o grau de erro intra-examinadores cometido durante dois momentos, com finalidade de se obter maior confiabilidade na totalidade dos traçados e das medidas obtidas.

A segunda etapa corresponde à análise estatística utilizada para avaliação das grandezas cefalométricas angulares e lineares utilizadas no presente estudo.

\section{Cálculo do erro}

Visando avaliar o erro do método na obtenção dos valores cefalométricos utilizados neste estudo, de forma a 


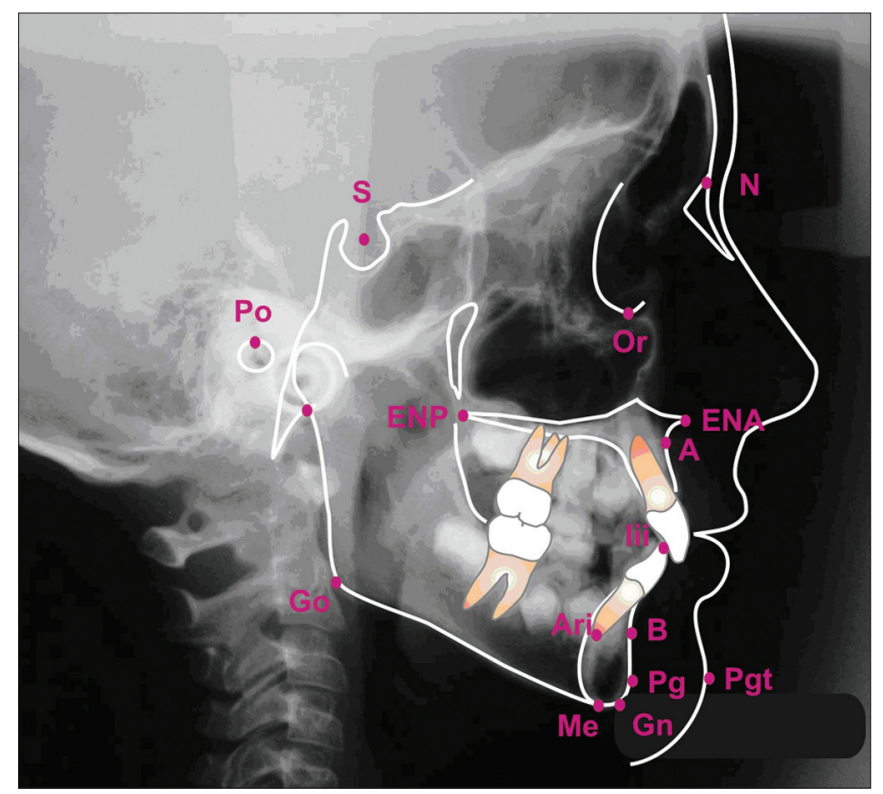

Figura 1. Pontos cefalométricos

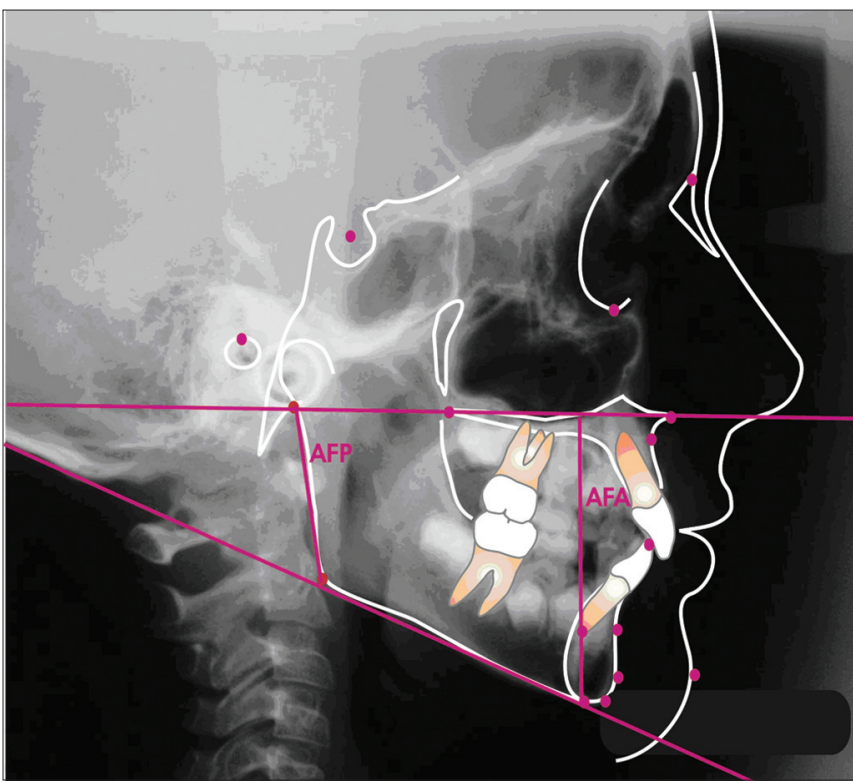

Figura 3. Grandezas lineares

AFP - altura facial posterior; AFA - altura facial anterior

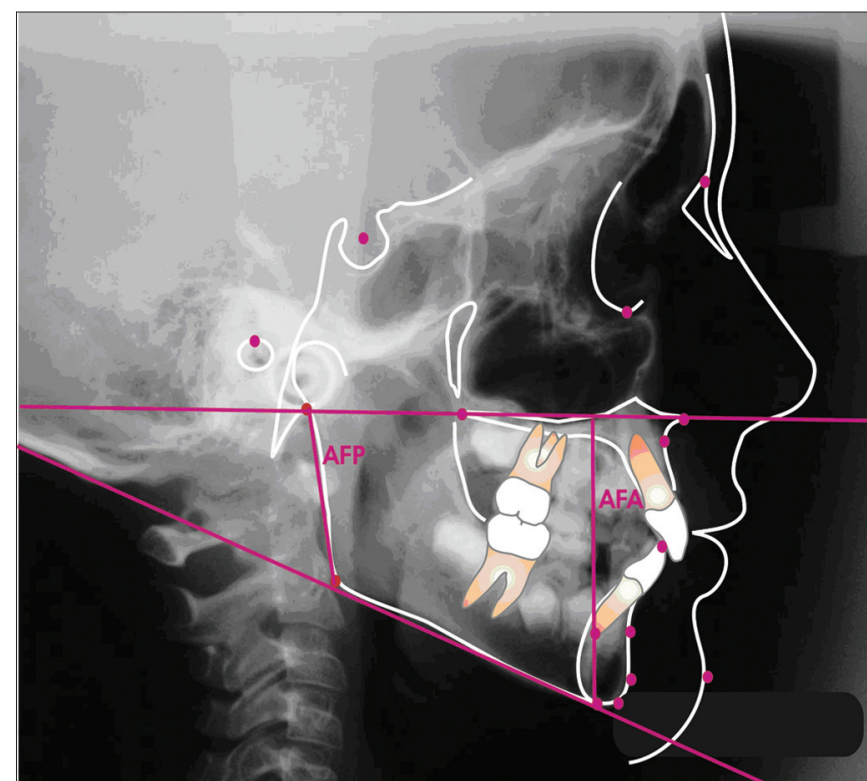

Figura 2. Traçado dos planos e linhas cefalométricos

\begin{tabular}{ll} 
1- Linha SN & 5- Plano Mandibular Go-Gn \\
2- Plano Horizontal de Frankfut & 6- Eixo Y \\
3- Plano Palatino & 7- Linha NA \\
4- Plano Mandibular Go-Me & 8- Linha NB \\
9- Longo eixo do incisivo inferior & 10- linha Z \\
\hline
\end{tabular}

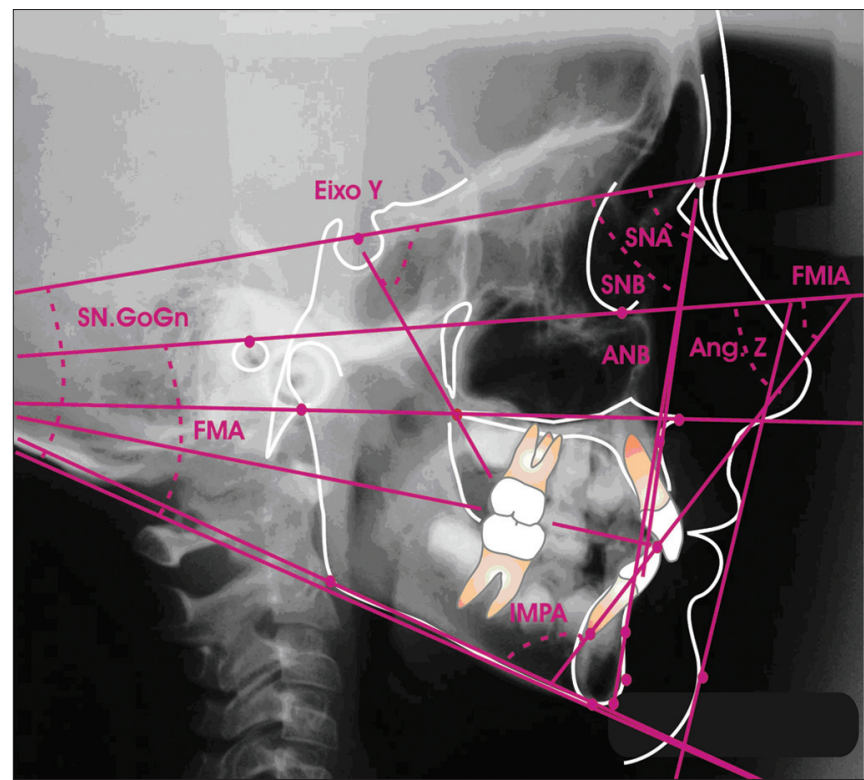

Figura 4. Grandezas angulares 
apurar os dados obtidos, aumentando-se a sua exatidão e confiabilidade, após 30 dias, realizaram-se repetições dos mesmos cefalogramas na totalidade.

Determinou-se o cálculo do erro de acordo com a fórmula proposta por Dalberg e defendida por Houston, em 1983, como se segue;

onde d é o desvio padrão da repetibilidade e:

sendo di o erro para o individuo i, o erro médio e n o número de indivíduos.

Foi ainda realizado um teste "t" pareado com nível de significância de 5\%, comparando-se os dois traçados.

De forma a apurar os dados obtidos, aumentando-se a sua exatidão e confiabilidade, cada telerradiografia foi traçada duas vezes pelo mesmo pesquisador, obtendo-se dois valores para cada variável cefalométrica. Por meio do cálculo de média aritmética simples, chegou-se a um valor médio e esse foi empregado no cálculo das grandezas esqueléticas da análise cefalométrica, de Merrifield \& Klontz (1993) e incluídos o ângulo SN-GoGn e o ângulo Y. Portanto avaliaram-se duas variáveis lineares, uma percentual e oito angulares.

Para a análise dos dados, obteve-se inicialmente uma análise descritiva (média e desvio padrão) e posteriormente aplicou-se o teste F e o teste "t" de Student com nível de significância de 5\%.

\section{RESULTADOS E DISCUSSÃO}

O cálculo do erro para as medidas duplicadas no grupo nasal está apresentado na Tabela 1. Observa-se que não ocorreu diferença significativa entre os traçados ( $p$ $>0,05)$, obtendo-se, assim, confiabilidade para os dados obtidos.

O cálculo do erro para as medidas duplicadas, no grupo bucal, está apresentado na Tabela 2; observa-se que não ocorreu diferença significativa entre os traçados ( $p$ $>0,05)$, obtendo-se, assim, confiabilidade para os dados obtidos.

A Tabela 3 apresenta a Média e desvio padrão para as grandezas e para o erro, bem como o teste ' $t$ " comparando os traçados nos dois momentos para os dois grupos.

As médias dos valores de 11 grandezas cefalométricas obtidas após a avaliação das 50 telerradiografias são apresentadas na Tabela 4. Observa-se não ocorreu diferença significante entre os grupos controle (respiração nasal) e o grupo experimental (respiração predominantemente bucal), $\mathrm{p}>0,05$.

As Figuras 5, 6 e 7, demonstram respectivamente os gráficos das variações angulares, lineares e percentuais dos dois grupos estudados.

A relação da função respiratória com o desenvolvimento da oclusão é um assunto polêmico. Autores como Ricketts (1968), Cooper (1989)1, Behlfelt et al. (1990),

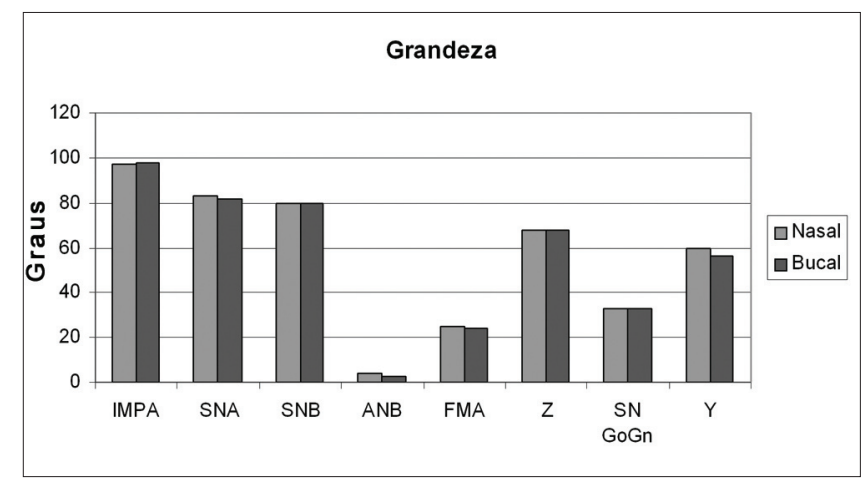

Figura 5. Média e desvio padrão das grandezas angulares para os grupos predominantemente nasal e bucal.

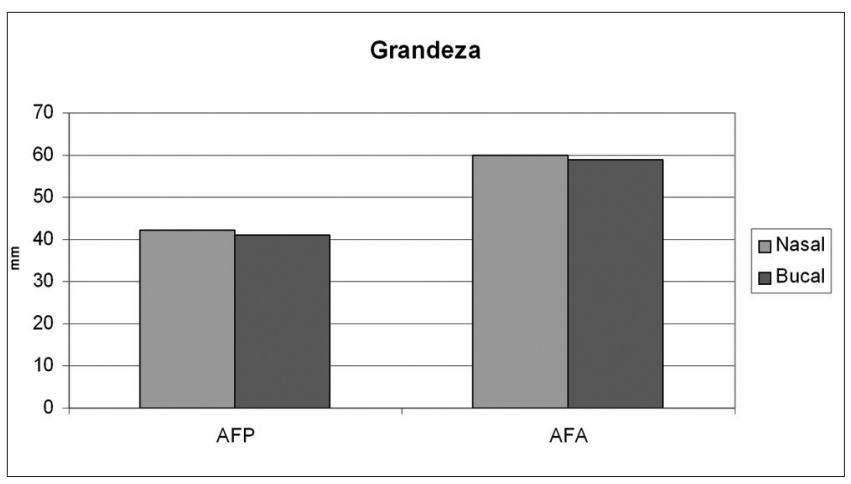

Figura 6. Média e desvio padrão das grandezas lineares para os grupos predominantemente nasal e bucal.

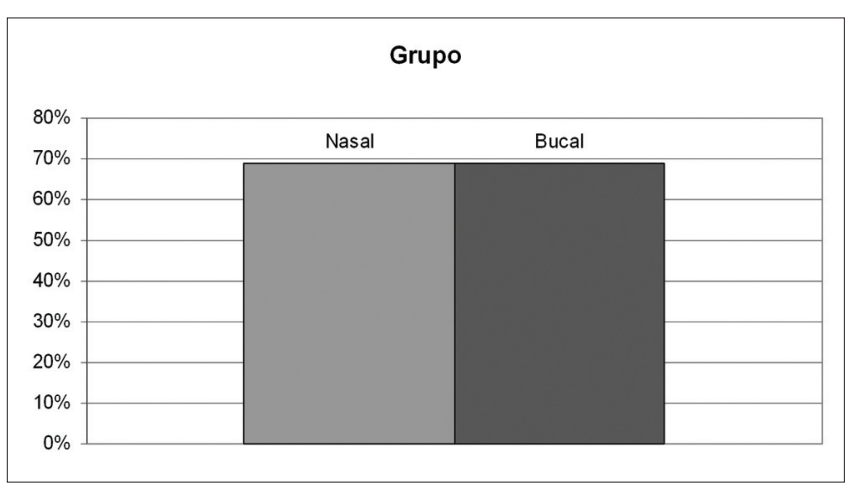

Figura 7. Média e desvio padrão da grandeza percentual, Índice de altura facial (IAF) para os grupos predominantemente nasal e bucal. 
Tabela 1. Média, desvio padrão e teste t comparando os traçados nos dois momentos para o grupo nasal.

\begin{tabular}{|c|c|c|c|c|c|c|c|}
\hline \multirow{2}{*}{ Grandezas } & \multicolumn{2}{|c|}{ Momento 1} & \multicolumn{2}{|c|}{ Momento 2} & \multicolumn{2}{|c|}{ Erro } & \multirow{2}{*}{ Teste $\mathrm{t}$} \\
\hline & Média & Desvio Padrão & Média & Desvio Padrão & Média & Desvio Padrão & \\
\hline IMPA & 99,52 & 5,13 & 99,12 & 4,99 & & 1,61 & 0,39 \\
\hline SNA & 83,00 & 3,30 & 82,88 & 3,70 & & 0,55 & 0,45 \\
\hline SNB & 80,06 & 3,14 & 79,82 & 3,36 & & 0,92 & 0,37 \\
\hline ANB & 2,98 & 1,21 & 2,88 & 1,28 & & 0,71 & 0,62 \\
\hline FMA & 25,04 & 3,42 & 24,96 & 3,19 & & 0,73 & 0,70 \\
\hline AFP & 41,48 & 2,54 & 41,56 & 2,58 & & 0,40 & 0,49 \\
\hline AFA & 59,08 & 3,43 & 58,96 & 3,72 & & 1,14 & 0,71 \\
\hline $\mathrm{IAF}$ & 69,74 & 4,40 & 69,72 & 4,40 & & 1,49 & 0,96 \\
\hline Z & 68,40 & 5,58 & 67,68 & 5,35 & & 1,94 & 0,20 \\
\hline SN-GoGn & 33,36 & 4,03 & 33,70 & 3,89 & & 0,77 & 0,13 \\
\hline$Y$ & 56,64 & 2,45 & 56,64 & 2,64 & & 0,89 & 1,00 \\
\hline
\end{tabular}

Fields et al. (1991) ${ }^{28}$, Mocellin (1992) e Fujiki \& Rossato $(1999)^{11}$ relacionam diretamente a respiração bucal como fator primário da maloclusão. Outros, como Emslie et al. (1952), Rubin (1980), Subtelny (1980) ${ }^{20}$, Harvold et al. $(1981)^{32}$, McNamara Jr. (1981) , Weber et al. (1981) Linder-Aronson \& Woodside (1982), Miller et al. (1982) ${ }^{42}$, Tomer \& Harvold (1982), Solow et al. (1984) ${ }^{43}$, Cheng et al. (1988) e Jabur (1997) relacionam a respiração bucal como um fator de desequilíbrio neuromuscular, que secundariamente poderia provocar ou mesmo intensificar uma maloclusão. A crítica literária quanto aos resultados encontrados nas pesquisas com respiradores predominantemente bucais é que na maioria das vezes o padrão respiratório é diagnosticado sem fundamentos científicos. Autores como Emslie et al. (1952), Massler \& Zwemer (1953) ${ }^{37}$, Diamond $(1980)^{19}$, Vig et al. $(1981)^{21}$, Klein (1986) $)^{24}$, Cheng et al. (1988), Cooper (1989) $)^{1}$, Smith \& Gonzales $(1989)^{26}$, Vig (1998), Crouse et al. (2000) $)^{44}$, Parolo \& Bianchini (2000), Queluz \& Gimenez (2000) $)^{2}$, Ianni Filho et al. (2001) ${ }^{36}$ e Jorge $(2001)^{35}$ concluem que a respiração bucal não pode ser, subjetivamente, diagnosticada. Trabalhos como os de Miller et al. (1982) ${ }^{42}$ e Tourne $(1990)^{27}$ chamam a atenção para os estudos que são realizados com seres humanos, as diferenças na anatomia da orofaringe e na musculatura dos macacos mostram que existe necessidade de cuidado na extrapolação dos dados em experimentos com esses animais para a população humana.

O objetivo do presente trabalho foi de comparar, cefalometricamente, respiradores nasais e predominantemente bucais. Neste contexto, seguindo a hipótese formulada, podemos notar, pelos resultados encontrados, que não ocorreram alterações estatisticamente significativas, determinadas pelo teste "t", a 5\%, para nenhuma das medidas estudadas quando comparados o grupo de respiradores nasais e grupo de respiradores predominantemente bucais.
Dessa forma, ao ser analisado o padrão de crescimento craniofacial determinado pelas grandezas FMA, SN-GoGn e ângulo Y, pode-se observar, pelos resultados encontrados, um padrão equilibrado para o grupo de respiradores nasais tanto quanto para o grupo de respiradores predominantemente bucais, ou seja, no grupo de respiradores nasais, o FMA avaliado na radiografia da cabeça em PNC apresentou um valor médio de 25,44 \pm 6,66, demonstrando um padrão mesofacial, embora com grande variabilidade demonstrado pelo valor elevado do desvio padrão . Por outro lado, quando comparados com o grupo de respiradores bucais 24,96 \pm 3,27 não foram observadas alterações estatisticamente significativas, comprovadas pelo teste "t" de student entre os dois grupos avaliados. Para a grandeza SN-GoGn o grupo de respiradores nasais apresentou um valor médio de 33,4 $\pm 4,39$, demonstrando um padrão mesofacial também com uma significativa variabilidade demonstrada pelo desvio padrão, Por outro lado, quando comparados com o grupo de respiradores bucais 33,79 \pm 4,57 não foram observadas alterações estatisticamente significativas, comprovadas pelo teste " $\mathrm{t}$ " de student entre os dois grupos avaliados.

O ângulo Y no grupo de respiradores nasais apresentou um valor médio de 59,16 \pm 3,25 e de 58,92 \pm 3,37 para os respiradores predominantemente bucais, demonstrando um padrão mesofacial também com uma significativa variabilidade demonstrada pelo desvio padrão. Para esta variável, quando comparados os dois grupos, não foram observadas alterações estatisticamente significativas comprovadas pelo teste "t" de student. Os resultados deste estudo não indicaram nenhuma diferença no padrão facial quando comparados os dois grupos, discordando com os resultados dos autores Koski \& Lähdemäki (1975), Schulhof (1978), Rubin (1980), Harvold et al. (1981)32, McNamara Jr (1981)7, Bresolin et al. (1983)8, Bressolin et al. (1984), 
Miller et al. (1984) ${ }^{34}$, Solow et al. (1984) ${ }^{43}$, Santos-Pinto \& Monnerat (1986) ${ }^{23}$, Melsen et al. (1987) , Cheng et al. (1988), Martins (1988), Jabur et al. (1997), Yamada et al. $(1997)^{10}$, Fujiki \& Rossato (1999) $)^{11}$, Motonaga et al. $(2000)^{14}$ e Pereira et al. (2001); e corroborando com os resultados dos autores Linder-Aronson \& Bäckström (1960), Quick \& Gundlach $(1978)^{18}$, Tarvonen \& Koski (1987), Smith \& Gonzales (1989) ${ }^{26}$, Bizetto (2000) ${ }^{13}$ e Mello (2001).

Com relação à avaliação do perfil facial determinado pelo ângulo Z, pode-se notar pelos resultados encontrados um valor diminuído tanto para o grupo de respiradores nasais quanto para o grupo de respiradores predominantemente bucais. O grupo de respiradores nasais apresentou um valor médio de $67,90 \pm 8,55$, sugerindo um perfil convexo com uma significativa variabilidade, demonstrada pelo alto desvio padrão. Por outro lado, quando comparados com o grupo de respiradores predominantemente bucais 68,4 $\pm 5,29$, também sugerindo um perfil convexo, não foram observadas alterações estatisticamente significativas, comprovadas pelo teste " $\mathrm{t}$ " de student, entre os dois grupos avaliados.

O perfil facial alterado pode estar relacionado à protrusão do incisivo inferior, que podemos notar pelos resultados encontrados num valor aumentado tanto para o grupo de respiradores nasais quanto para o grupo de respiradores predominantemente bucais. O grupo de respiradores nasais apresentou um valor médio de 98,32 $\pm 4,57$, que é maior do que a média considerada normal, e uma significativa variabilidade demonstrada pelo alto desvio padrão. Contudo, quando comparados com o grupo de respiradores bucais 99,26 \pm 4,82, também sugerindo uma vestibularização, não foram observadas alterações estatisticamente significativas, comprovadas pelo teste "t" de student. O resultado encontrado para essa grandeza corrobora com Smith \& Gonzales (1989) ${ }^{26}$ e diferem dos resultados de alguns autores como Hawkins (1969)5, McNamara Jr $(1981)^{7}$, Bresolin et al. (1983) ${ }^{8}$, Bressolin et al. (1984), Santos-Pinto \& Monnerat (1986) ${ }^{23}$, Cheng et al. (1988), Behlfelt et al. (1990), Fields et al. (1991) ${ }^{28}$, Mocelin $(1992)^{4}$, Marchesan et al. (1995), Mocellin \& Ciuffi (1997), Fujiki \& Rossato (1999) $)^{11}$, Motonaga et al. (2000) $)^{14}$, e Pereira et al. (2001), que encontraram um perfil mais convexo nos respiradores predominantemente bucais quando comparados com os respiradores nasais.

Quanto ao relacionamento das bases ósseas com a base do crânio, avaliada pelas grandezas, SNA, SNB e ANB respectivamente, observou-se pelos resultados encontrados para os respiradores nasais um ângulo SNA de $83,54 \pm 2,91$, valor ligeiramente aumentado, porém dentro da variação do padrão de normalidade (82). Para o grupo de respiradores bucais, o ângulo SNA apresentou um valor médio de 82,84 \pm 3,46, também dentro da variação do padrão de normalidade. Quando comparamos o ângulo SNA do grupo de respiradores nasais com o ângulo SNA do grupo de respiradores predominantemente bucais, não foram observadas alterações estatisticamente significativas, comprovadas pelo teste "t" de student.

O valor obtido para o ângulo SNB foi de 79,86 \pm 3,12 para os respiradores nasais, estando dentro da variação do padrão de normalidade (80). Para o grupo de respiradores predominantemente bucais o ângulo SNB apresentou um valor médio de 79,96 \pm 3,20, também dentro da variação do padrão de normalidade. Por outro lado, quando comparados o grupo de respiradores nasais com o grupo de respiradores predominantemente bucais, não foram observadas alterações estatisticamente significativas, comprovadas pelo teste "t" de student.

O valor obtido para a grandeza ANB foi de 3,49 \pm 1,02 para os respiradores nasais, classificando o grupo como Classe I esquelética. Para o grupo de respiradores bucais, o ANB apresentou um valor médio de 2,98 \pm 1,21, também dentro da variação do padrão de Classe I. Por outro lado, quando comparados o grupo de respiradores nasais com o grupo de respiradores predominantemente bucais não foram observadas alterações estatisticamente significativas, comprovadas pelo teste "t" de student. Os resultados obtidos no presente estudo são contrários aos resultados dos autores Hawkins (1969)5, Paul \& Nanda (1973), Harvold et al. (1981) 32, Bressolin et al. (1984), Santos-Pinto \& Monnerat (1986) ${ }^{23}$, Melsen et al. (1987) , os quais encontraram um aumento do ANB descaracterizando o padrão de Classe I (ANB variando de 1 a 5). Contudo, os resultados encontrados no presente trabalho corroboram com os autores Linder-Aronson \& Bäckström (1960), Watson et al. (1968), Muñoz (1970), Carbone \& Bernaba (1977), Schulhof (1978) e Smith \& Gonzales (1989) ${ }^{26}$ que também não encontraram nenhuma diferença estatisticamente significativa no relacionamento das bases ósseas.

Para a altura facial posterior, determinada pela grandeza linear AFP, observa-se, pelos resultados encontrados, um valor diminuído tanto para o grupo de respiradores nasais, quanto para o grupo de respiradores predominantemente bucais. O grupo de respiradores nasais apresentou um valor médio de 41,80 $\pm 3,16$, sugerindo uma falta de crescimento da altura do ramo, com uma média variabilidade demonstrada pelo desvio padrão. No grupo de respiradores predominantemente bucais, a AFP apresentou um valor de 41,66 \pm 2,88, também sugerindo uma falta de crescimento da altura do ramo, com uma média variabilidade demonstrada pelo desvio padrão. Por outro lado, quando comparados entre si, não foram observadas alterações estatisticamente significativas, comprovadas pelo teste "t" de student, entre os dois grupos avaliados.

A altura facial anterior, determinada pela grandeza linear AFA, apresentou um valor diminuído tanto para o grupo de respiradores nasais, quanto para o grupo de respiradores predominantemente bucais. O grupo de respiradores nasais apresentou um valor médio de 59,72 \pm 
3,97, sugerindo uma diminuição da distância vertical entre o plano palatino e o mento, com uma alta variabilidade demonstrada pelo valor do desvio padrão. No grupo de respiradores predominantemente bucais, a AFA apresentou um valor de 59,84 \pm 4,00, também sugerindo uma diminuição da distância vertical entre o plano palatino e o mento, com uma alta variabilidade demonstrada pelo valor do desvio padrão. Por outro lado, quando comparadas entre si, não foram observadas alterações estatisticamente significativas, comprovadas pelo teste " $\mathrm{t}$ " de student, entre os dois grupos avaliados.

$O$ índice da altura facial, determinado pela grandeza IAF, demonstra uma AFP em relação AFA dentro da variação considerada equilibrada para os dois grupos em estudo, apesar de uma alta variabilidade demonstrada pelo valor alto do desvio padrão, com um valor de 69,72 \pm 6,45 para o grupo de respiradores nasais e de 69,36 \pm 6,58 para o grupo de respiradores nasais (padrão de normalidade IAF $=69 \%$ ). O IAF entre os dois grupos avaliados, quando comparados entre si, não apresentou alterações estatisticamente significativas, comprovadas pelo teste "t" de student. Os resultados obtidos no presente estudo corroboram com os autores Quick \& Gundlach (1978) ${ }^{18}$, Linder-Aronson (1979) ${ }^{6}$ e Smith \& Gonzales (1989) ${ }^{26}$ e são contrários aos resultados dos autores Harvold et al. $(1973)^{31}$, Linder-Aronson (1979) $)^{6}$, Bressolin et al. (1984), Santos-Pinto \& Monnerat (1986) ${ }^{23}$, Melsen et al. (1987) ${ }^{9}$ e Martinez Esteinou \& Omana Vidal (1988), os quais encontraram um aumento da altura facial anterior, levando também a uma alteração no índice da altura facial.

Constatamos que não ocorreram alterações estatisticamente significativas entre os dois grupos comparados nas grandezas consideradas no presente estudo, confirmando a preocupação de que a respiração bucal não pode ser considerada com fator primário de maloclusão para esta amostra em questão.

\section{CONCLUSÃO}

Com base nos resultados obtidos, pode-se concluir que:

a) Não ocorreu diferença estatisticamente significativa quando se avaliaram o padrão facial para ambos os grupos (meso-facial), representados pelas grandezas: FMA, SN-GoGn e ângulo do eixo Y;

b) Considerando os perfis faciais, caracterizados pelo ângulo Z, na amostra estudada, os dois grupos se comportaram de forma estatisticamente semelhante (perfil convexo);

c) Quanto ao relacionamento entre as bases apicais, representado pelas grandezas SNA, SNB, ANB, pode-se observar que não ocorreram alterações estatisticamente significativas, assim como para as alturas faciais posteriores e anteriores.

d) Não ficou comprovada, entre os grupos respira- dores nasais e predominantemente bucais, a existência de diferenças estatisticamente significativas entre as grandezas avaliadas, portanto, com base nos resultados do presente trabalho, concluímos que a respiração bucal nem sempre pode ser considerada como um único agente etiológico de modificação do padrão facial.

e) Como o estudo possui características transversais sugerem-se novos trabalhos longitudinais.

\section{REFERÊNCIAS BIBLIOGRÁFICAS}

1 Cooper BC. Nasorespiratory function and orofacial development. Otolaryngol Clin North Am 1989; 22(2):413-41.

2 Queluz DP, Gimenez CMM. A síndrome do respirador bucal. Rev CROMG. 2000; 6(1):4-9.

3 Andrade LP, Majolo MS. A influência da respiração bucal no crescimento facial. Rev Goiana Ortod 2000; 5:34-45.

4 Mocellin M. Respirador bucal. In: Petrelli E. Ortodontia para fonoaudiologia. São Paulo: Lovise; 1992. p.131-4.

5 Hawkins AC. Mouth breathing and its relationship to malocclusion and facial abnormalities. N M Dent J. 1969; 20(1):18-21.

6 Linder-Aronson S. Respiratory function in relation to facial morphology and the dentition. Br J Orthod 1979; 6(2):59-71.

7 McNamara JA. Influence of respiratory pattern on craniofacial growth. Angle Orthod 1981; 51(4):269-300.

8 Bresolin D, Shapiro PA, Shapiro GG, Chapko MK, Dassel S. Mouth breathing in allergic children: its relationship to dentofacial development. Am J Orthod 1983; 83(4):334-40.

9 Melsen B, Attina L, Santuari M, Attina A. Relationships between swallowing pattern, mode of respiration, and development of malocclusion. Angle Orthod 1987; 57(2):113-20.

10 Yamada T, Tanne K, Miyamoto K, Yamauchi K. Influences of nasal respiratory obstruction on craniofacial growth in young Macaca fuscata monkeys. Am J Orthod Dentofacial Orthop. 1997; 111(1):38-43.

11 Fujiki PDT, Rossato C. Influência da hipertrofia adenoideana no crescimento e desenvolvimento craniodentofacial. Ortodontia. 1999; 32(1):70-9.

12 Sabatoski CV. Estudo comparativo das dimensões esqueléticas verticais e horizontais entre crianças respiradoras bucais e nasais [dissertação] Curitiba: Centro de Ciências Biológicas e da Saúde, Pontifícia Universidade Católica do Paraná; 1999. 96 f.

13 Bizetto MSP. Estudo cefalométrico vertical comparativo entre crianças com respiração bucal e nasal nos diferentes tipos faciais [dissertação]. Curitiba: Pontifícia Universidade Católica do Paraná; 2000. 105f.

14 Motonaga SM, Berti LC, Anselmolima WT. Respiração bucal: causas e alterações no sistema estomatognático. Rev Bras Otorrinolaringol 2000; 66(41):373-9.

15 Simas Netta MLS, Maruo H, Vieira SR, Saga AY. Estudo cefalométrico comparativo das dimensões craniofaciais entre crianças respiradoras nasais e bucais, com maloclusão Classe II divisão 1. J Brás Ortod Ortop Facial 2004; 9(49):41-7.

16 Lusvarghi L. Identificando o respirador bucal. Rev Assoc Paul Cir Dent 1999; 53(4):265-74.

17 Klein E. Obstrução nasal: um obstáculo à vida. Rev Bras Otorrinolaringol. 1987; 53(4):106-10.

18 Quick CA, Gundlach KK. Adenoid facies. Laryngoscope 1978; 88(2 Pt 1):327-33.

19 Diamond O. Tonsils and adenoids: why the dilemma? Am J Orthod 1980; 78(5):495-503.

20 Subtelny JD. Oral respiration: facial maldevelopment and corrective dentofacial orthopedics. Angle Orthod 1980; 50(3):147-64.

21 Vig PS, Sarver DM, Hall DJ, Warren DW. Quantitative evaluation of nasal airflow in relation to facial morphology. Am J Orthod 1981; 79(3):263-72.

22 O'Ryan FS, Gallagher DM, LaBanc JP, Epker BN. The relation between nasorespiratory function and dentofacial morphology: a review. Am 
J Orthod 1982; 82(5):403-10.

23 Santos-Pinto A, Monnerat ME. Alterações nasofaringeana e crânio-faciais em pacientes com adenóide hipertrófica: estudo cefalométrico. RGO. 1986; 34(4):349-54.

24 Klein JC. Nasal respiratory function and craniofacial growth. Arch Otolaryngol Head Neck Surg. 1986; 112(8):843-9.

25 Meredith GM. Airway and dentofacial development. Folha Med 1988; 97(1):33-40.

26 Smith RM, Gonzalez C. The relationship between nasal obstruction and craniofacial growth. Pediatr Clin North Am 1989; 36(6):1423-34.

27 Tourne LP. The long face syndrome and impairment of the nasopharyngeal airway. Angle Orthod 1990; 60(3):167-76.

28 Fields HW, Warren DW, Black K, Phillips CL. Relationship between vertical dentofacial morphology and respiration in adolescents. Am J Orthod Dentofacial Orthop 1991; 99(2):147-54.

29 Castilho JCM, Generoso R, Moraes LC, Moraes MEL. Avaliação radiográfica da altura facial anterior inferior (AFAI) em pacientes com e sem obstrução da nasofaringe. J Bras Ortod Ortop Facial 2002; 7(38):133-41.

30 Solow B, Greve E. Rhinomanometric recording in children. Rhinology 1980; 18(1):31-42.

31 Harvold EP, Vargervik K, Chierici G. Primate experiments on oral sensation and dental malocclusions. Am J Orthod 1973; 63(5):494-508.

32 Harvold EP, Tomer BS, Vargervik K, Chierici G. Primate experiments on oral respiration. Am J Orthod 1981; 79(4):359-72.

33 Weber ZJ, Preston CB, Wright PG. Resistance to nasal airflow related to changes in head posture. Am J Orthod 1981; 80(5):536-45.

34 Miller AJ, Vargervik K, Chierici G. Experimentally induced neuromuscular changes during and after nasal airway obstruction. Am J Orthod 1984; 85(5):385-92.

35 Jorge EP. Estudo da resistência nasal em pacientes com má-oclusão de Classe II divisão $1^{\underline{a}}$ de Angle, utilizando a rinomanometria anterior ativa. Rev Dent Press Ortod Ortop Facial 2001; 6(1):15-30.

36 Ianni Filho D, Raveli DB, Raveli RB, Castro Monteiro Loffredo L, Gandin Jr LG. A comparison of nasopharyngeal endoscopy and lateral cephalometric radiography in the diagnosis of nasopharyngeal airway obstruction. Am J Orthod Dentofacial Orthop 2001; 120(4):348-52.

37 Massler M, Zwemer JD. Mouthbreathing. II. Diagnosis and treatment.
J Am Dent Assoc 1953; 46:658-71.

38 Quinn GW. Airway interference syndrome. Clinical identification and evaluation of nose breathing capabilities. Angle Orthod 1983; 53(4):311-9.

39 Thuer U, Kuster R, Ingervall B. A comparison between anamnestic, rhinomanometric and radiological methods of diagnosing mouthbreathing. Eur J Orthod 1989; 11(2):161-8.

40 Chami FAI. Avaliação nasofibroscópica e radiológica de pacientes com hiperplasia da amigada faríngea. Rev Bras Med. 1998; 5(4):118-24.

41 Krogman WM, Sassouni V. A syllabus in roentgenographic cephalometry. Philadelphia: Philadelphia Growth Study; 1957. p. 45-103.

42 Miller AJ, Vargervik K, Chierici G. Sequential neuromuscular changes in rhesus monkeys during the initial adaptation to oral respiration. Am J Orthod 1982; 81(2):99-107.

43 Solow B, Siersbaek-Nielsen S, Greve E. Airway adequacy, head posture, and craniofacial morphology. Am J Orthod 1984; 86(3):214-23.

44 Crouse U, Laine-Alava MT, Warren DW. Nasal impairment in prepubertal children. Am J Orthod Dentofacial Orthop. 2000; 118(1):69-74.

45 Gross AM, Kellum GD, Michas C, Franz D, Foster M, Walker M et al Open-mouth posture and maxillary arch width in young children: a three-year evaluation. Am J Orthod Dentofacial Orthop. 1994; 106(6):635-40.

46 Hartgerink DV, Vig PS. Lower anterior face height and lip incompetence do not predict nasal airway obstruction. Angle Orthod 1989; 59(1):17-23.

47 Jacobson A. The "Wits" appraisal of jaw disharmony. Am J Orthod 1975; 67(2):125-38

48 Marchesan IQ, Krakaver LH. A importância do trabalho respiratório na terapia miofuncional. Tópicos em Fonoaudiologia. São Paulo: Lovise; 1995 . v.2, p.155-60.

49 Negreiros PE. O efeito sobre as medidas cefalométricas devido à alteração da posição natural da cabeça (PNC) em telerradiografias tomadas em norma lateral [dissertação]. Piracicaba: Faculdade de Odontologia de Piracicaba, Universidade Estadual de Campinas; 2000.

50 Oliveira RC, Anselmo-Lima WT, Souza BB. Importância da nasofibroscopia na presença do RX cavum normal para diagnóstico da hiperplasia adenoideana. Rev Bras Otorrinolaringol 2001; 67(4 pt1):499-505. 